\title{
Condensed Matter involving Dark Matter and Negative Mass
}

\author{
By N. SCOTT \\ May 12,2021 \\ nikolascott97@gmail.com
}

\section{. Abstract}

The question that has bewildered the physics community is the colossal complexities of the idea of three categorical similarities dependent on each other. Dark Energy, Dark Matter and Anti-Gravity. One could say that Anti-Gravity is actually dark matter. In other words, they are one in the same. Based on my theory, all three of these similarities are connected via a formula I extended from the Potential Energy formula into a new, original formula. Is this the beginning of a quantization of a form of Matter that has never been quantified?

\section{. Part I}

Potential energy measured in joules is calculated by the following formula. $P E=m g h$. This is energy that is based off of the height according to the ground. But what if we were able to reverse this? Into something that requires more energy. I mean dark energy. This is the formula I have devised to explain such a thing.

[eq. 1.]

$$
\text { Anti }- \text { Gravity } P E=m g(R-h)
$$


And if you know that $R$ is the radius of the Earth you can now imagine and visually see how much minimum energy would be needed for Anti-Gravity. Now taking the limit from $\left(A G P E \lim _{h \rightarrow R}\right)$. You could then perceive that once the height reaches the radius of the Earth the value converges to zero. Imagine the value of what $(R-h)$ could be. Knowing compared to $R$, $h$ must be very small but it must be included for scientific accuracy. Now let's take the equation further into space. If you were to go further from Earth say $1 A U=h$, then unless the mass of the object is negative the energy will be negative beyond Earth. Which brings me to another formula I devised.

Knowing $R$, [eq. 2.]

Dark Potential Energy $=$ mass of object ${ }^{*} g *($ Radius of Earth $-(h$ in Universal Range

Which if outside of Earth will always be a large amount of dark energy. This isn't to say dark energy couldn't be obtained on Earth.. because it can. If you were to assume the Anti-Gravity formula since we are describing a calculation on earth. Knowing [eq. 1.] if mass were to be negative, then the energy would become dark energy. This is the only theoretical way to obtain dark energy on Earth. Though [eq. 2.] clearly concludes why many scientists have assumed that $2 / 3 \mathrm{rds}$ of the Universe is dark energy. And this is due to [eq. 2.] 
What is negative mass precisely? You could say it is dark matter itself. It would only make sense for the creation of dark energy would involve an ingredient we all know as dark matter. This only makes logical sense.

Shortening [eq. 2.]

Dark Potential Energy $=m g($ Radius of Earth $-(h$ in Universal Range $)$

Note: This dark energy is in conjunction with positive matter. As in they coexist with each other.

..First Conclusion

Potential use cases for dark matter:

The first thing that comes to fruition is the thought of spaceships. If one were to apply my Anti-Gravitational law into place and somehow obtain negative-mass "dark matter" then the race for a spaceship could be no further from an impossibility. All it would take would be a simple model spacecraft that involves dark matter, otherwise without the negative-mass it would not work.

Law of Anti-Gravity: Anti-Gravity can only be affected by dark matter. If one were to acquire negative-mass objects one could use the $A G P E=m g(R-h)$ formula to identify how much dark energy would be necessary for 
Anti-Gravitational flight.

..Second Conclusion(further explanation)

Making it to the Moon was a great feat. made by the United States of American organization known as NASA. Much has been discovered by the rocket scientists of the twentieth century. But something has been missing in rocket science. An explanation for Dark Energy and Dark Matter. The laws of Newtonian bodies have yet to provide an explanation for.. Or the concept of a body free from gravity. Now object-free-gravity or OFG does not exclude the orbital part of the Earth into the equation. As the Orbital has its own value in the OFG equation. Thus you could call it the OFG equation as thus stated. [eq. 3.]

$$
O F G_{\text {Orbital }}=m g(R-(h=R)
$$

[eq. 4.]

$$
O F G_{O r b}=m g(O r b)
$$

This incites the idea that once the Orbital is reached, zero gravity exists, but not OFG. OFG exists beyond and before Orb. Now the initial location to start would be ground level when working with the OFG field equation.

As in you would start it this way, [eq. 5.] 


$$
O F G_{P E \text { grounded }}=m g(h=0)
$$

[eq. 6.]

$$
O F G_{P E G}=m g(g n d)
$$

Now you could also say at the grounded level there is no potential energy to exist. You're going to have to use two different equations to separate OFG and PEG, then stating equation 7 .

[eq. 7.]

$$
P E G_{0}=P E G_{g n d} \text { equal to } \mathrm{mg}(\mathrm{h}=\odot) \text { while }
$$

[eq. 8.]

$$
O F G_{0}=m g(O r b)
$$

As such, these are two completely different bodies in relation to gravity. As the gnd value for $h$ is zero energy. Meanwhile the $\operatorname{Orb}$ value for $h=R-R$ is object-free-energy or OFG. Now OFG is always free of gravity but in the Orbital it turns into free fall or weightlessness. OFG is only weightless before the orbital area and beyond it. This must be considered.

As OFG on the ground is different from PEG on the ground.

Given the equation,

[eq. 9.] 


$$
O F G=m g(R-h) \text { is not the same as }
$$

[eq. 10.]

$$
P E G=m g h
$$

The reason being that when $h$ is zero for OFG the amount of Dark Energy available or required at $h=0$ is equal to $m g R$ precisely. This is different from PEG, which is equal to absolutely zero energy available or required. Which means PEG's formula is useless at $h=0$, but OFG is not useless at all. It gives a definite answer of $m g R$ energy necessary for object-free-gravity. In other words, it is the most, but only on Earth. Note this though. OFG doesn't work unless there is negative mass for the object. In space positive mass will be acquainted with dark matter and dark energy. Yet on Earth dark matter is the negative mass and produces dark energy. Giving us the equation:

[eq. 11.]

$$
\begin{gathered}
-O F G=-m g(R-h) \text { the dark matter-Earth-correlation } \\
\text { or DEC. }
\end{gathered}
$$

Thus, you could say

[eq. 12.]

$$
-O F G=D E C
$$

And...

[eq. 13.] 


$$
D E C=D M g(R-h) \text { while DM is Dark Matter }
$$

So, if you put any value for $h \rightarrow R$, you will notice that for example.

If $D M=1$, then $D E C=1 \mathrm{~g}(\mathrm{R}-\mathrm{h})$, thus, if $\mathrm{DM}>1$ then $D E C>D E C$ where $D M=1$. Meaning the Dark Energy will only increase with the increase in Dark Matter, which makes logical sense.

..Third Conclusion(space)

Knowing all of the previous equations there is one other equation to explain. And that is the Universal-h-equation or UHE

The UHE is of the following form:

[eq. 14.]

$$
U H E=m g(R-h \text { in Universal Range })
$$

The first thought some might think is, what does this entail? Well there is only one way to explain it entirely. And that is by correlating UHE with Dark Energy, Dark Matter and Positive Matter, or DE, $D M$ \& $P M$. Now $-D M=P M$ \& $-P M=D M$. Knowing this we could put these into the UHE.

[eq. 15.]

$$
U H E=-D M(R-h \text { in } U E R)
$$

Meaning the further into space according to any Radius coordinate on Earth results and ends in UER-end. Or UEReP. 
"Universal-energy-radius-end-point." And with this end is where the most of the Dark Energy resides. Say for instance, you took one energy-mass-point EMP equal to one UEReP. Then,

[eq. 16.]

$$
E M P=U E R e P \text { then, }
$$

$[\mathrm{eq} \cdot 17$.

$$
E M P_{n}=U_{E R e}
$$

And ultimately,

[eq. 18.]

$$
U E R e P_{n}=U H E_{\max }
$$

Or in simpler terms,

[eq. 19.]

$$
\operatorname{UEReP}_{n}=D E_{\max }
$$

Or, the maximum amount of Dark Energy to exist in the Universe, being based on the number of Universal-energy-radius-points with their energies added together to form $D E_{\max }$. A further analysis could imply that $D E_{\max }$ is a certain percentage of the Universal-energy UE. Knowing this one could then make a full UE equation by the following: [eq. 20.]

$$
U E=D E_{\max }+P E_{\max }, P E \text { being Positive Energy }
$$


Going back to energy-mass-points, [eq. 21.]

$$
E M P=-D M(R-\text { Maximum of } A U U R)
$$

Whereas, AU is astronomical units and UR is Universal Radius, assuming the Universe is finite, and it is theorized by most but ever-expanding. Meaning the amount of Dark Energy could be expanding in acceleration.

We know that (R-Maximum of $A U$ UR) is going to be a very large negative number. We also know that Dark Matter with a negative mass multiplied by this, is equal to One EMP energy-mass-point. Though, we also know that Dark Matter with a positive mass produces Dark Energy. Meaning Dark Matter can be both large and small, confirming the ideas and theories most of which either of whom thought Dark Matter was very massive or very small. This proves that it is rather both, massive and small.

\section{..Final Conclusion}

Now calculating the amount of Dark Energy could also be quantified with all of this information. The formula would be:

[eq. 22.]

$$
E M P_{n}=\operatorname{UEReP}\left(R_{n}\right) \text { where } R \text { is the radius of the Earth. }
$$


So multiplying the finite amount of radii on Earth globally is equal to the number of energy-mass-point-ends that exist.

So,

This would amount for $100 \%$ of the more than or equal to amount of $2 / 3$ rds theorized Dark Energy that exists in the entire Universe. Whereas the left indicates the number of energy-mass-points, the right supports this with by multiplying the number of Earth radii by one UEReP, Universal-energy-Radius-end-point.

..Extended Consciousness-Added-Chapter

There must be a correlation from Schumann Frequency ELF "Extremely Low Frequency" bands, Dark Matter and humans. However Dark Matter affects us must be equal to the effect of the Schumann Resonances' quantified affect on humans undoubtedly. Therefore, asking how a distributed frequency such as the Schumann frequency is the same as asking how distributed DM affects us in this case, you could see that for each end point of Schumann, is equal for all DM points.

[eq. 22.]

$$
S(1)=D M(1)
$$

Does this mean the consciousness of one human being is equal to $1 / S(1)_{n}$ or $1 / D M(1)_{n}$, meaning the size in energy of a human's consciousness is equal to this? 
Now if you were to combine all consciousness of the Earth, the combined energy might even be equal to $D M(1)_{n}$ or $S(1)_{n}$. Does this mean human consciousness has just been measured for the first time? Maybe not completely but it is a start. If HConsc is equal to a certain negative mass, is that mass a certain portion of the Universe? Can it be more than equal to or less than another consciousness. Possibly there are those that possess more Dark Energy than others. At a minimum of $1 / D M(1)_{n}$ and with a maximum Dark Energy of DM(1) sub $n$.

The question is.. were all consciousness equal at some point? Doubtful. It seems the god or gods have given some more power than others. In ancient scripts that is to say. How can one obtain more Dark Energy for themselves? Should one subvert themselves to a long exposure to powerful frequencies to make themselves as strong as gods? Is there limitless Dark Energy due to the ever-expanding Universe. If so, what is causing it to expand? Is it a few individuals trying to grant themselves power over others? Were they given dormant power to begin with that they would unlock anyway at some point? If this is the case, then transcendental beings are special in this way. Meaning there are only very few who possess the power to increase their own power. And this select few actually have a Destiny. 
[1.] Google. (n.d.). From MACHOs to WIMPs-meet the top five candidates for 'dark matter'. Google. https://www.google.com/amp/s/phys.org/news/2015-12-m achos-wimpsmeet-candidates-dark.amp.

[2.] Garner, R. (2020, September 10). Hubble Observations Suggest Missing Ingredient in Dark Matter Theories. NASA.

https://www. nasa.gov/feature/goddard/2020/hubble-obs ervations-suggest-a-missing-ingredient-in-dark-matte r-theories.

..Declarations

Funding: Not applicable.

Conflicts of interest/Competing interest: Not applicable.

Availability of data and material: Not applicable.

Code availability: Not applicable.

Authors'contributions: Not applicable.

Ethics approval: Not applicable.

Consent to participate: Not applicable.

Consent to publication: Not applicable. 